Noname manuscript No.

(will be inserted by the editor)

\title{
Dynamic Multilateral Markets
}

\author{
Arnold Polanski · Emiliya Lazarova
}

the date of receipt and acceptance should be inserted later

\begin{abstract}
We study dynamic multilateral markets, in which players' payoffs result from intra-coalitional bargaining. The latter is modeled as the ultimatum game with exogenous (time-invariant) recognition probabilities and unanimity acceptance rule. Players in agreeing coalitions leave the market and are replaced by their replicas, which keeps the pool of market participants constant over time. In this infinite game, we establish payoff uniqueness of stationary equilibria and the emergence of endogenous cooperation structures when traders experience some degree of (heterogeneous) bargaining frictions. When we focus on market games with different player types, we derive, under mild conditions, an explicit formula for each type's equilibrium payoff as the market frictions vanish. JEL classification: C72, C78, J30.
\end{abstract}

Keywords multilateral bargaining $\cdot$ dynamic markets

\section{Introduction}

Markets where the terms of trade are negotiated among several participants abound in modern economies. Joint ventures, (financial) intermediation or complex global supply chains, are examples of partnerships that typically involve more than two parties. ${ }^{1}$ We offer a theoretical framework which is well suited to study how the strategic behavior of players leads to the formation of prices in such multilateral markets. This framework allows us to derive various properties of equilibrium prices and compare them to the benchmark represented by competitive prices.

In our setup, we adapt Rubinstein and Wolinsky (1985)'s seminal model of a strategic buyer-seller market to fit multilateral environments. The market consists of a set of participants and at every date non-intersecting subsets of these players meet with an exogenously given probability. Upon meeting, a coalition of players has the possibility to generate surplus by employing their members' inputs. Coalition members need to first

\footnotetext{
We are indebted to Helmuts Azacis, Daniel Cardona, Corrado Di Maria, Alex Gershkov, Rob Gilles, Fernando Vega-Redondo, participants at ASSET 2010, CTN 2011 meetings, York Annual Symposium on Game Theory 2011, Game Theory World Congress 2012, EEA-ESEM Meeting 2012 and seminar participants at Queen's University Belfast, University of Manchester and University of East Anglia, two anonymous referees and the associate editor for helpful feedback and constructive comments.

Address(es) of author(s) should be given

${ }^{1}$ Multi-party coalitions, committees, and multinational alliances are but a few other environments where decisions are reached via multilateral negotiations.
} 
agree on how to divide the surplus amongst themselves. The division of the surplus defines the terms of trades or prices awarded to the inputs held by individual members. If an unanimous agreement is reached, it is binding, and players trade, leave the market, and are replaced by replica players. Otherwise, all players stay on the market and new subsets of market participants are randomly selected to meet.

We note that our market game is of complete but imperfect information as we assume that players do not observe the terms of agreements they are not part of. ${ }^{2}$ One way to analyze this game is to follow, e.g., Rubinstein and Wolinsky (1985), and define its extensive form. This definition specifies histories and strategies for all players. In particular, for any agent, the history at a certain stage of the game is the sequence of observations made by her up to that stage. A strategy for any agent is, then, a sequence of decision rules conditional on all histories that dictates player's moves, i.e., the offers she makes as a proposer and her acceptance/rejection responses to offers made to her. Rubinstein and Wolinsky (1985) focus on stationary strategies. They define market equilibrium as a stationary strategy profile such that no agent can improve by changing her action after any possible history.

To determine equilibrium payoffs we employ the notion of stationary market equilibrium which is also used by Rubinstein and Wolinsky (1985). Like Gul (1989), Chatterjee et al. (1993), and more recently Yan (2009) we find that stationarity strengthens the predictive power of the equilibrium. ${ }^{3}$ In fact, we show the payoff uniqueness of the stationary equilibria in all games in which players exhibit some positive degree of impatience (Theorem 1).

To analyze in greater detail the properties of equilibrium payoffs, we extend our analysis to market games where players are characterized by their types and own type-specific factors of production. Here, we focus on the limit behavior of players, i.e. we study stationary equilibria when players become infinitely patient. In this environment, we show that players of the same type receive identical limit equilibrium payoffs, i.e., that prices are anonymous; and that these payoffs exhaust the value of those coalitions which reach an agreement (Theorem 2, items (i) and (ii)). This result implies the endogenous emergence of unique limit equilibrium price for each factor of production. Furthermore, we establish that each factor of production in a limit market equilibrium is rewarded (approximately) its marginal contribution to the coalition where agreement is reached (Theorem 2, item (iii)). As such, unlike Gul (1989) and Hart and Mas-Collel (1996a), we find that the limit market equilibria of our market game are unrelated to the Shapley value of the corresponding transferrable utility game. Moreover, in Theorem 3 we fully characterize players' payoffs in the limit partitioning equilibrium. In such an equilibrium, each player type cooperates in at most one coalition type, i.e., the set of player types is partitioned into subsets that cooperate in non-intersecting coalitions. In Theorem (4), we derive a condition on the primitives of the market game that ensures that the unique equilibrium is partitioning. Finally, we discuss a continuous approximation of our market game and illustrate it with an application to labor markets with heterogenous workforce.

With its focus on dynamic multilateral bargaining, this paper contributes to two growing branches of literature: that on dynamic markets (for example, in the context of seller-buyer markets see Rubinstein and Wolinsky (1985), Binmore and Herrero (1988), Rubinstein and Wolinsky (1990), and, more recently Duffie et al. (2005) and Manea (2011)); and that on coalitional bargaining (e.g. Chatterjee et al. (1993), Hart and Mas-Collel (1996a), Chatterjee and Sabourian (2000), and more recently Okada (2011)). Based on its bargaining procedure, our work is closest in nature to Nguyen (2011), Siedlarek (2012) and Manea (2011). In fact, our Theorem 1 can be seen as a generalization of Manea's result on the payoff-uniqueness when the matched bilateral coalitions are network links with normalized values. We show that this uniqueness holds also in bargaining games on weighted networks and, more generally, in games on hypergraphs that represent

\footnotetext{
2 This assumption is innocuous for our results that focus on stationary equilibria.

3 The multiplicity of perfect equilibria in the extension of Rubinstein (1982) model to multilateral bargaining has been first pointed out by Shaked and reported by Sutton (1986).
} 
multilateral coalitions. ${ }^{4}$ Our contribution lies further in characterizing the unique equilibrium payoffs in the limit. In particular, we derive an explicit functional form of the limit payoffs when the limit equilibrium satisfies what we call the partitioning property. We furthermore provide a characterization of the market games where this partitioning property holds.

Our work differs from other studies on multilateral markets in the bargaining procedure and matching mechanism. Several other works study multilateral bargaining, however, they do not consider a dynamic market. Some of these focus on characteristic function form games. These authors either aim at supporting core allocations as equilibrium outcomes (cf. Yan (2003)) or focus on the efficiency of the bargaining outcomes (cf. Chatterjee et al. (1993) and Okada (1996)). In a related contribution, Krishna and Serrano (1996) allow for "partial agreement" where the agreeing agents leave the bargaining procedure with their agreed shares, while the proposer and the disagreeing agents proceed to the next stage of bargaining. While the assumption of "partial agreement" may be valid in certain contexts, e.g., division of an estate, it is less applicable to others such as production with complementary inputs or formation of coalitions where all members are needed for the coalition to win a vote. Chatterjee and Sabourian (2000), instead, study unanimous agreement of all parties in the multi-person ultimatum game. They, however, assume that bargaining continues until agreement is reached. As our focus is on anonymous dynamic markets, the assumption that the bargaining coalition dissolves in case of disagreement seems more plausible. Finally, Okada (2011) studies coalitional bargaining in the context of coalition formation where a proposal is a pair of a coalition of players and a payoff vector.

The rest of the paper is organized as follows: In Section 2, we describe our theoretical framework and in Section 3 we characterize the stationary equilibrium in a market game. In Section 4, we specialize our discussion by introducing heterogeneous player types. There, we develop the notions of limit equilibrium and of partitioning limit equilibrium and we discuss their characterization. Finally, we exemplify a continuous approximation of the market game and illustrate it in a stylized labor market with heterogeneous workers.

\section{Market Game}

The market operates over an infinite number of discrete dates and is populated by a finite $\operatorname{set} \mathcal{N}$ of $N=|\mathcal{N}|$ agents. Each date in the market starts with a matching stage, in which some non-intersecting coalitions of players (subsets of $\mathcal{N}$ ) are randomly matched. The probability $\pi_{S}$ of selecting the coalition $S \subseteq \mathcal{N}$ is implied by an exogenous and time-invariant matching procedure $\mathcal{M}$. The latter is defined as a probability distribution over all collections of mutually non-intersecting subsets of $\mathcal{N}$.

The members of any matched coalition $S$ can produce a value $v(S) \geq v(\emptyset)=0$ by employing their player-specific inputs. In general, the value will reflect the coalition's productivity, possibly a function of complementarities across input types. ${ }^{5}$ The production of $v(S)$ can only take place if all members of $S$ agree $^{6}$ on the terms of trade. The latter are negotiated in the ultimatum bargaining game with recognition probabilities that are parameterized by the vector of strictly positive (absolute) bargaining powers $\boldsymbol{\alpha}=$ $\left(\alpha_{1}, \ldots, \alpha_{N}\right)$. The recognition probability of any replica of $i \in S$, conditioned on $S$ being matched, is then given by $\alpha_{i} / \alpha(S)$, where $\alpha(S):=\sum_{k \in S} \alpha_{k}$.

\footnotetext{
${ }^{4}$ In a concurrent work Nguyen (2011) takes convex analysis approach to study existence in coalition bargaining problem with network constraints.

5 We explore such setup in Section 4

${ }^{6}$ As argued in the Introduction, the requirement of unanimity of agreement may be justified by input complementarity and is a natural assumption in a voting environment where only minimal winning coalitions have a positive probability of being selected. Certainly, this assumption is not innocuous. For example, it drives the result that when players are impatient ( $\delta$ is small) unproductive players may obtain strictly positive payoffs in equilibrium.
} 
The intra-coalitional bargaining proceeds as follows: The selected proposer $i \in S$ makes sequentially (in any order) offers to the players in $S \backslash\{i\}$. If an offer is rejected by at least one responder, coalition $S$ dissolves immediately and the same population of players proceeds to the next date. ${ }^{7}$ Otherwise, there is an agreement, in which case the value $v(S)$ is created, agents in $S \backslash\{i\}$ receive their agreed shares and $i$ obtains the residual surplus. Then, the members of $S$ leave the market and are replaced by their replicas with the same endowments before the game moves to the next date. Therefore, the replica set $\mathcal{N}$ remains in the market throughout the entire history of the game. Importantly, all new agents are treated by the matching procedure in the same way as the ones who left. For example, the set of newcomers that have replaced the members of an agreeing coalition $S$, will be selected with probability $\pi_{S}$ in all subsequent periods, in which they stay in the market. ${ }^{8}$ Finally, we assume that each replica of player $i$ discounts future dates with the same factor $\delta_{i} \in(0,1)$. We collect the discount rates in the vector $\boldsymbol{\delta}=\left(\delta_{1}, \ldots, \delta_{N}\right)$. Some of our results hold in the limit when $\delta_{i}$ approaches one for all $i \in \mathcal{N}$. We shall interpret this situation as vanishing market frictions.

The market game is fully specified by its primitives $\mathcal{N}, \mathcal{M}, v(),. \boldsymbol{\alpha}$ and $\boldsymbol{\delta}$ and the above rules. In the next section, we characterize stationary equilibria of this game.

\section{Stationary Equilibria of the Market Game}

As much of the literature, we shall consider only stationary subgame perfect equilibria (SE). Therefore, we omit the formalization of histories and history-dependent strategies. ${ }^{9}$ At any date $t$, a stationary strategy of proposer $i \in S$ in a matched coalition $S$ will depend exclusively on the identities of responders in $S \backslash\{i\}$. Responder $j \in S \backslash\{i\}$, on the other hand, will condition her stationary strategy on the offer made to her. It follows that for a stationary strategy profile, matching environment, fixed discount factors, and bargaining powers, each replica of player $k \in \mathcal{N}$ expects the same payoff $x_{k}$ at the beginning of any date. In addition, we recall that the market game is of complete information. Thus, we can use backward induction to identify a subgame-perfect Nash equilibrium strategy at every subgame by eliminating all non-credible moves. We exploit these facts to derive conditions for the SE payoffs $\left\{x_{k}\right\}_{k \in \mathcal{N}}$. Specifically, when

$$
v(S)-\sum_{j \in S \backslash\{i\}} \delta_{j} x_{j}>\delta_{i} x_{i}, \text { or, equivalently, } v(S)>\sum_{j \in S} \delta_{j} x_{j},
$$

then the unique SE strategy is constructed as follows: if $j \in S \backslash\{i\}$ is the last responder in the matched coalition $S$, then she will accept in equilibrium any offer (weakly) larger than her continuation payoff, $\delta_{j} x_{j}$, and reject any offer below $\delta_{j} x_{j}$. Similarly, if the last but one responder, $k \in S \backslash\{i\}$, anticipates the acceptance by $j$, she will accept any offer (weakly) larger than her respective continuation payoff, $\delta_{k} x_{k}$, and reject any offer below $\delta_{k} x_{k}$. This argument propagates to all responders in $S \backslash\{i\}$. Note that these offers will be accepted with certainty as, otherwise, $i$, who earns the residual surplus $v(S)-\sum_{j \in S \backslash\{i\}} \delta_{j} x_{j}$, would increase them infinitesimally to ensure all sequential acceptances. If, on the other hand,

$$
v(S)=\sum_{j \in S} \delta_{j} x_{j}
$$

\footnotetext{
7 As all coalitional members need to agree for a proposal to be carried out, the proposer makes an offer of what Huang (2002) refers to as conditional nature.

${ }^{8}$ Similar assumptions are made by Manea (2011). Alternatively, given market fundamentals, we can focus on steady states in which, by definition, this condition is also satisfied.

9 Such formalization would be a straightforward generalization of that in Manea (2011) to coalitions with more than two members.
} 
a multiplicity of equilibrium strategies arises. For example, all responders in $S$ may be offered their respective discounted continuation payoffs and randomize their agreements. The multiplicity holds also when,

$$
v(S)<\sum_{j \in S} \delta_{j} x_{j}
$$

In this case, the proposer will not make an acceptable offer to all the responders because she anticipates that such an offer may be accepted, in which case her own payoff would be below her continuation payoff. Hence, an agreement is never reached. ${ }^{10}$ Importantly for our purposes, the responders in a matched coalition obtain their continuation payoffs in any SE. Therefore, the conditions on the SE payoffs can be succinctly captured by the following system of $N$ equations,

$$
\begin{aligned}
x_{i} & =\sum_{S \in S_{i}} \pi_{S}\left(\frac{\alpha_{i}}{\alpha(S)} \max \left\{v(S)-\sum_{j \in S \backslash\{i\}} \delta_{j} x_{j}, \delta_{i} x_{i}\right\}+\frac{\left(1-\alpha_{i}\right)}{\alpha(S)} \delta_{i} x_{i}\right)+\left(1-\sum_{S \in S_{i}} \pi_{S}\right) \delta_{i} x_{i} \\
& =\delta_{i} x_{i}+\sum_{S \in S_{i}} \pi_{S} \frac{\alpha_{i}}{\alpha(S)} \max \left\{v(S)-\sum_{j \in S} \delta_{j} x_{j}, 0\right\}, \quad \forall i \in \mathcal{N},
\end{aligned}
$$

where $S_{i}$ is the set of all coalitions $S \subseteq \mathcal{N}$ containing player $i \in \mathcal{N}$. A solution $\mathbf{x}^{\delta}=\left(x_{i}^{\delta}\right)_{i \in \mathcal{N}}$ to the system (1) yields the expected SE payoffs. A unique solution, in particular, yields unique payoffs although these payoffs can be supported by multiple strategy profiles. Recall that multiplicity occurs, for example, when responder $j$ in the matched coalition $S$, for which it holds $v(S)=\sum_{i \in S} \delta_{i} x_{i}$, agrees with any probability to the offer $\delta_{j} x_{j}$.

Given our assumption that replica agents trade only once, we can interpret the payoff $x_{i}$ as the price that player $i$ expects for her unit of input in an SE. One can also consider an alternative model where agents enter the market with a stock of inputs. In such a case the disagreement payoffs should internalize the utility loss due to delayed trade of all remaining units of the input. This will make the relation of equilibrium payoffs to input unit prices less straightforward to interpret.

The following discussion illustrates the nature of equilibrium prices in several stylized markets.

Example 1 We consider a market with three players, $\mathcal{N}=\{1,2,3\}$, and discuss several cases of value functions that capture various bargaining scenarios. To simplify our discussion and notation, in each case we only list the values of the productive coalitions which have a positive probability to meet; in other words, we assume $v(S)=0$ or $\pi_{S}=0$ for all unlisted coalitions $S \subseteq \mathcal{N} .{ }^{11}$ In addition, we will assume that all productive coalitions are matched with equal probabilities and that the sum of these probabilities is one.

Bilateral Bargaining First, we consider a simple game of one-buyer-one-seller bargaining where $v(\{1,2\})=$ 1 (i.e., here, we take player 3 to be unproductive). Then, the unique solution to the system (1) yields the SE payoffs,

$$
x_{1}^{\delta}=\frac{\alpha_{1}\left(1-\delta_{2}\right)}{\alpha_{1}\left(1-\delta_{2}\right)+\alpha_{2}\left(1-\delta_{1}\right)}, \quad x_{2}^{\delta}=\frac{\alpha_{2}\left(1-\delta_{1}\right)}{\alpha_{1}\left(1-\delta_{2}\right)+\alpha_{2}\left(1-\delta_{1}\right)} .
$$

\footnotetext{
${ }^{10}$ Notice that when the value of the coalition is less than the total discounted continuation payoffs of its members, $v(S)<$ $\sum_{j \in S} \delta_{j} x_{j}$, and, therefore, there is a responder $j$ who is made an offer which is below her continuation payoff, then all responders preceding $j$ are indifferent between accepting and rejecting their offers. This is because these players anticipate the rejection of $j$ of her offer. Thus, there are other SE strategies that are, however, payoff equivalent, as they all result in the disagreement of all coalitions $S$ for which $v(S)<\sum_{j \in S} \delta_{j} x_{j}$.

${ }^{11}$ For example, in the following discussion of the two-sided market, we do not list the value of the grand coalition, $v(\{1,2,3\})$. There we implicitly assume that the buyer never meets with the two sellers simultaneously, i.e, $\pi_{\{1,2,3\}}=0$.
} 
Clearly a player's equilibrium payoff is increasing in her relative bargaining power, $\frac{\alpha_{1}}{\alpha_{2}}$, and own discount factor.

In the following discussion, we will further assume that players have equal discount factors, $\delta_{i}=\delta$, and equal bargaining powers $\alpha_{i}=\alpha$ for all $i=1,2,3$.

Consider a two-sided market in which a single buyer (player 1) bargains with two identical sellers (players 2 and 3) over the price of one unit of a homogenous good. In particular, if we take $v(\{1,2\})=$ $v(\{1,3\})=1$, we obtain that the unique SE solution,

$$
x_{1}^{\delta}=\frac{2}{4-\delta}, \quad x_{2}^{\delta}=x_{3}^{\delta}=\frac{1}{4-\delta},
$$

illustrates the fact that the single buyer cannot extract the entire surplus from the competing sellers for any level of market frictions given by $\delta .^{12}$

Multilateral bargaining Similar to the simple bilateral example discussed first, when only the three-player coalition is productive, $v(\mathcal{N})=1$, the outcome of the game with equal bargaining powers is the equal division of the surplus, i.e., 1/3 for each player, irrespective of the discount factor $\delta$.

A non-trivial multilateral bargaining may be captured by $v(S)=1$ for all $S \subseteq \mathcal{N}$ with at least two members. Then, the unique solution to the system (1) for $\delta>4 / 5,{ }^{13}$

$$
x_{i}^{\delta}=\frac{1}{2(2-\delta)}, \quad i=1,2,3,
$$

implies an agreement by any matched pair of players and a disagreement in the grand coalition. This example shows also that the SE payoffs can differ from the Shapley values $(1 / 3,1 / 3,1 / 3)$ of the corresponding static transferrable utility game and that they are not in the core of this game, which, in this case is empty. This is driven by the fact that, as illustrated here, it is not necessary for the grand coalition to reach an agreement in a SE even if the matching technology is such that the grand coalition meets with a positive probability.

Notably, in all market games, discussed in the above example, a unique equilibrium price vector is obtained. The next result generalizes this observation to all multilateral bargaining games.

Theorem 1 There exists a unique SE payoff profile (i.e., a unique solution to the system (1)) in any multilateral bargaining game.

An elegant proof of this result in a slightly different set-up can be found in Nguyen (2011) (Theorem 1 there). Independently of the work of this author, we developed an alternative proof which can be found in the attached additional material. The main idea of the proof in Nguyen (2011) is to interpret the conditions for $\mathrm{x}^{\boldsymbol{\delta}}$ to be stationary equilibrium payoffs as a first-order condition of a convex program. Our proof strategy follows a different approach: first we replace in the system (1) each max operator by a product of the first argument of this operator with an agreement probability. In this way, we obtain a system of linear equations and show that this new system has a unique solution. Then, we define a correspondence that computes agreement probabilities that ensure optimal (dis)agreements, given some agreement probabilities and the

\footnotetext{
12 Polanski and Vega-Redondo (2013) extend this example further and study market participation by heterogeneous buyers and sellers.

13 For $\delta<4 / 5$ the solution $x_{i}^{\delta}=\frac{4}{3(4-\delta)}$ to (1) implies an agreement in all matched coalitions. For $\delta=4 / 5$, we have equilibrium agreements with certainty in all coalitions of size two, and with any probability in the grand coalition.
} 
solution to the linear system for the latter probabilities. We show that this correspondence has a fixed point, which implies the existence of a solution to (1). Finally, we show that the existence of two different SE payoff profiles induced by two different sets of rationally agreeing coalitions leads to a contradiction.

\section{Limit Equilibria and Multilateral Coalitions}

So far we have conducted our analysis without imposing any restriction on the characteristic value function, and we have derived our existence and uniqueness result in this very general setup. In order to derive sharper predictions on the structure of equilibrium payoff profiles, we further assume that players can be partitioned into types. One can view players of the same type as agents who own one unit of the same homogeneous input. Players of different types, on the other hand, are distinguished by the different characteristics of their productive inputs. Specifically, we consider a partitioning of the set of agents $\mathcal{N}$ into $T$ types, i.e., $\mathcal{N}=\cup_{t=1, \ldots, T} \mathcal{N}_{t}$ and $\mathcal{N}_{s} \cap \mathcal{N}_{t}=\varnothing$ for all $s, t=1, \ldots, T$ with $s \neq t$. For all types $t=1, \ldots, T$, we denote the cardinality of the set $\mathcal{N}_{t}$ by $N_{t}$. A multilateral coalition (MC) $S \subseteq \mathcal{N}$ consists of $\sum_{t=1, \ldots, T} n_{t}$ players, where $n_{t}=\left|S \cap \mathcal{N}_{t}\right|$ denotes the number of $t$-type players in this coalition. The type of any MC coalition $S \subseteq \mathcal{N}$ is given by the ordered vector of type multiplicities, $\boldsymbol{n}=\left(n_{1}, \ldots, n_{T}\right)$ such that $n_{t}=\left|S \cap \mathcal{N}_{t}\right|$ denotes the number of $t$-type players in this coalition, and we denote by $\mathbf{n}^{\mathcal{N}}$ the set of all possible coalition types in the set $\mathcal{N}$. We also use the operator $\boldsymbol{T}(S): \mathcal{S} \rightarrow \mathbf{n}^{\mathcal{N}}$, where $\mathcal{S}=\{S \mid S \subseteq \mathcal{N}\}$, to obtain the type profile of any MC coalition $S \subseteq \mathcal{N}$. For example, the grand coalition is of type $\boldsymbol{T}(\mathcal{N})=\left(N_{1}, \ldots, N_{T}\right)$, while a singleton coalition $\{i\}$ with $i \in \mathcal{N}_{t}$ is of type $\boldsymbol{T}(\{i\})=\mathbf{e}_{t}$, where $\mathbf{e}_{t}$ is the canonical unit vector that points in direction $t$.

We make the natural assumption that the set of coalition types maps onto the set of coalition values, and, therefore coalitions of the same type have the same productivity, i.e., $v(S)=v\left(S^{\prime}\right)$ when $\boldsymbol{T}(S)=\boldsymbol{T}\left(S^{\prime}\right)$. Therefore, with a slight abuse of notation, we will adopt the practice to denote by $v(\boldsymbol{T}(S))$ the productivity of coalition $S$ which is of type $\boldsymbol{T}(S)$. Furthermore, we assume that each productive MC $S$ has a positive probability of meeting, $\pi_{S}>0$ if $v(S)>0$. Finally, a symmetric treatment of players of the same type requires that such players have equal absolute bargaining powers and discount factors, $\alpha_{i}=\alpha_{j}$ and $\delta_{i}=\delta_{j}$ if $\boldsymbol{T}(\{i\})=\boldsymbol{T}(\{j\})$. In order to avoid technicalities, we will further assume equal discount factors $\delta_{t}=\delta$ for players of all types $t=1, \ldots, T$, and derive asymptotic results when $\delta$ converges to one. To facilitate further the exposition, we also adopt the shorthand notation $x(S)=\sum_{i \in S} x_{i}$. Our aims are to characterize agreements in coalitions of different types and derive explicit expressions for the players' limit equilibrium payoffs. Formally, a limit equilibrium is defined below.

Definition 1 The vector $\mathrm{x}^{1}:=\lim _{\delta \rightarrow 1} \mathrm{x}^{\delta}$, where $\delta=(\delta, \ldots, \delta)$ and $\mathrm{x}^{\delta}$ is a solution to (1) will be called a limit $S E(L E)$.

In Lemma 1 (in the Appendix), we show that the limit $\mathbf{x}^{1}$ is well defined and that there is a threshold $\underline{\delta}<1$ such that for all $\delta \in(\underline{\delta}, 1)$ the collection of coalitions that (dis)agree with certainty in equilibrium is the same. The agreeing coalitions will be called active in the LE $\mathrm{x}^{1}$. The set of active coalitions defines the cooperation structure of LE. Furthermore, we will say that player $i$ cooperates in the LE $\mathbf{x}^{1}$ if there exists a coalition $S$ of which $i$ is a member, $i \in S$, and $S$ is active in $\mathbf{x}^{1}$.

The following result establishes payoff-equivalence among players of the same type, the value exhaustion for each active MC and the bounds on the LE payoffs.

Theorem 2 Consider a market game and its $L E \mathbf{x}^{1}$. Then,

(i) all players of type $t=1, \ldots, T$, receive the same payoff $x_{t}^{1}$; 
(ii) for each coalition of type $\mathbf{n}=\left(n_{1}, \ldots, n_{T}\right)$, it holds that

$$
\sum_{s=1}^{T} n_{s} x_{s}^{1} \geq v(\mathbf{n})
$$

where equality holds for each active coalition.

(iii) a player of type $t$ that cooperates in the LE in coalitions of type $\mathbf{n}$ receives (approximately) her marginal contribution to this type of coalitions,

$$
v\left(\mathbf{n}+\mathbf{e}_{t}\right)-v(\mathbf{n}) \leq x_{t}^{1} \leq v(\mathbf{n})-v\left(\mathbf{n}-\mathbf{e}_{t}\right),
$$

provided $v\left(\mathbf{n}+\mathbf{e}_{t}\right)$ and $v\left(\mathbf{n}-\mathbf{e}_{t}\right)$ are well-defined.

All proofs can be found in the appendix.

Theorem 2 carries straightforward implications for the LE payoff of unproductive agents. ${ }^{14}$ Formally, agents' type $t=1, \ldots, T$ is defined as dummy type if for all MC coalition types $\mathbf{n} \subseteq \mathbf{n}^{\mathcal{N}}$ where $n_{t}>0$, we have $v(\mathbf{n})=v\left(\mathbf{n}-\mathbf{e}_{t}\right)$. Then the following corollary follows immediately from property (iii) of Theorem 2 .

Corollary 1 In any LE the payoff of all agents of dummy type is zero.

Next, we discuss a simple voting game that illustrates the use of Theorem 2 .

Example 2 First, we recall that in an $N$-player apex game the value of coalition $S \subseteq \mathcal{N}$ is,

$$
v(S)= \begin{cases}1, & i \in S \text { and } S \backslash\{i\} \neq \emptyset \\ 1, & S=\mathcal{N} \backslash\{i\} \\ 0, & \text { otherwise. }\end{cases}
$$

Player $i$ is called the main, or apex, player. As all but the apex player are symmetric w.r.t. the function $v($.$) ,$ there are only two types of players in this game: the single apex player (type 1 ) and $N-1$ type 2 players, where $N=|\mathcal{N}| \geq 2$.

By Theorem 2 property (ii) and the coalition value function (3), the total payoff for the coalitions with type profiles $(1, k), k=1, \ldots, N-1$, and $(0, N-1)$ must satisfy,

$$
x_{1}^{1}+k x_{2}^{1} \geq 1, \quad(N-1) x_{2}^{1} \geq 1 .
$$

We note that the second inequality in (4) implies that $x_{2}^{1}>0$ and, hence, the first inequality must be strict when $k>1$. Hence, there are only two possible active profiles $(1,1)$ and $(0, N-1)$ in the LE of the apex game. Moreover, as we show in the discussion of Example 3 below, both coalition types must be active in the LE when $\alpha_{1}=\alpha_{2}$. Then, the LE payoffs follow by property Theorem 2 property (ii),

$$
\begin{aligned}
x_{1}^{1}+x_{2}^{1} & =1 \quad \& \quad(N-1) x_{2}^{1}=1 \Rightarrow \\
x_{1}^{1} & =\frac{N-2}{N-1}, \quad x_{2}^{1}=\frac{1}{N-1} .
\end{aligned}
$$

In order to refine our results further we focus on a special type of LE that we shall call partitioning. Intuitively, LE is partitioning if each player type cooperates in at most one coalition type, i.e., the set of player types is partitioned into subsets that cooperate in non-intersecting coalitions. Formally,

\footnotetext{
14 We thank one anonymous referee for pointing this out.
} 
Definition $2 A L E \mathrm{x}^{1}$ is partitioning $(P E)$ if,

$$
\forall S, S^{\prime} \subseteq \mathcal{N}: S \cap S^{\prime} \neq \varnothing, \quad S, S^{\prime} \text { active in } \mathrm{x}^{1} \Rightarrow \boldsymbol{T}(S)=\boldsymbol{T}\left(S^{\prime}\right)
$$

In a PE, two distinct coalitions that contain at least one agent of the same type are both active if these coalitions are of the same type. Partitioning equilibria will arise naturally in many games, e.g., when only one type of coalition is productive as in the homogenous buyer-seller market discussed in Example 1. One can easily construct examples, however, where the unique LE is not partitioning. For instance, the LE in a two-sided market with heterogenous buyers/sellers will not be usually partitioning. The next theorem fully characterizes the payoffs in an PE as functions of fundamentals.

Theorem 3 In the $P E \mathbf{x}^{1}$, all players of type $t=1, \ldots, T$, who cooperate in coalitions of type $\mathbf{n}$, receive

$$
x_{t}^{1}=v(\mathbf{n}) \frac{n_{t} \alpha_{t} / N_{t}}{\sum_{s=1}^{T}\left(n_{s}^{2} \alpha_{s} / N_{s}\right)} .
$$

The relative PE payoffs depend, therefore, on bargaining powers $\alpha_{1}, \ldots, \alpha_{T}$, stocks $N_{1}, \ldots, N_{T}$ and demands $n_{1}, \ldots, n_{T}$ of types in the equilibrium cooperation structure. A notable common feature of the results summarized in Theorems 2 and 3 is that they are independent of the matching procedure provided that each productive coalition is matched with a positive probability. A sufficient and necessary condition for the partitioning property of the LE is stated in our next result.

Theorem 4 For a type profile $\mathbf{t}=\left(t_{1}, \ldots, t_{T}\right)$ and a set $P \subseteq\{1, . ., T\}$, let $\mathbf{t}^{P}$ be a type profile such that $\mathbf{t}_{i}^{P}=t_{i}$ if $i \in P$ and $\mathbf{t}_{i}^{P}=0$ otherwise. Then, the unique LE in the market game is partitioning if and only if there is a type profile $\mathbf{n}$ and a partition $\mathcal{P}$ such that

$$
\sum_{P \in \mathcal{P}} v\left(\mathbf{n}^{P}\right) \sum_{t \in P} \frac{m_{t} n_{t} \alpha_{t} / N_{t}}{\sum_{s \in P} \alpha_{s} n_{s}^{2} / N_{s}}>v(\mathbf{m}), \quad \forall \mathbf{m} \notin\left\{\mathbf{n}^{P}\right\}_{P \in \mathcal{P}}, \mathbf{m} \neq \mathbf{0} .
$$

If the above condition holds, then $\mathbf{n}^{P}$ is the type of active coalitions for the player types in $P$. Note that we do not require that the profile $\mathbf{n}^{P}$ is unique. This simplifies the verification of (7) as in many cases there will be natural candidates for active profiles. Below, we illustrate the use of condition (7) to show that the apex game (see Example 2) is not partitioning.

Example 3 Recall that in Example 2, we established that in an apex game, there are exactly two types of players: type 1 is the type of the apex player, and type 2 is the type of any other player. Hence, there are only two possible partitions of the type set: $\mathcal{P}_{1}=\{\{1,2\}\}$ and $\mathcal{P}_{2}=\{\{1\},\{2\}\}$. For $\mathcal{P}_{1}$, consider coalitions with type profiles $\mathbf{n}=\left(n_{1}, n_{2}\right), \mathbf{m}=\left(m_{1}, m_{2}\right)$ with $v(\mathbf{n})=v(\mathbf{m})=1$ and $\alpha_{1}=\alpha_{2}$. Then condition (7) takes the form,

$$
m_{1} n_{1}+m_{2} n_{2} /(N-1)>n_{1}^{2}+n_{2}^{2} /(N-1), \quad \forall \mathbf{m} \neq \mathbf{n}, \mathbf{m} \neq \mathbf{0}
$$

This condition is, however, violated for $\mathbf{m}=(1,1)$ if $\mathbf{n} \neq(1,1)$, and, for $\mathbf{m}=(0, N-1)$ if $\mathbf{n}=(1,1)$.

Next consider $\mathcal{P}_{2}$ and type profiles $\mathbf{n}=\left(n_{1}, n_{2}\right), \mathbf{m}=\left(m_{1}, m_{2}\right)$ with $v(\mathbf{n})=v(\mathbf{m})=1$ and $\alpha_{1}=\alpha_{2}$. Then, condition (7) takes the form,

$$
v\left(\left(0, n_{2}\right)\right) m_{2}>n_{2}, \quad \forall \mathbf{m} \notin\left\{\left\{n_{1}, 0\right\},\left\{0, n_{2}\right\}\right\}, \mathbf{m} \neq \mathbf{0} .
$$

This condition is, however, violated for $\mathbf{m}=(1,1)$ as $v\left(\left(0, n_{2}\right)\right)=1$ if $n_{2}=N-1$ and $v\left(\left(0, n_{2}\right)\right)=$ 0, otherwise. It follows, therefore, that the apex game is not partitioning: some players will be active in coalitions of at least two different types. 


\section{Continuous Approximation to a Labor Market}

This section introduces a continuous approximation of the market game discussed so far. We apply this technique to the study of a labor market game and compare our results to some finings in the literature on firm-worker bargaining. Importantly, our discussion can be viewed as providing an alternative wage-setting mechanism to the tâtonnement process of competitive equilibrium. By adopting a continuous approximation method our analysis is simplified, which further allows us to focus on the comparative statics of our results. In a concluding remark, however, we draw parallels with the discrete case scenario.

In the approximation, we shall assume that the function $v: A \rightarrow R$, where $A=\prod_{t=1 \ldots, T}\left[0, N_{t}\right]$, is differentiable and increasing in each argument. As in e.g. Hart and Mas-Collel (1996b), we will interpret the arguments of $v($.$) as total masses of each of the T$ types. Similarly, $x_{s}^{1}$ will be interpreted as the LE price rate of type $s$. Thus, $m_{s} x_{s}^{1}$ is the total price for the mass $m_{s}$ of $s$-type players.

The properties (ii) and (iii) as stated in Theorem 2 for the LE $\mathrm{x}^{1}$ take then the form,

$$
\begin{aligned}
(i i)^{\prime} \quad \sum_{s=1}^{T} m_{s} x_{s}^{1} & \geq v(\mathbf{m}), \\
(i i i)^{\prime} \quad v\left(\mathbf{n}+\epsilon \mathbf{e}_{t}\right)-v(\mathbf{n}) & \leq \epsilon x_{t}^{1} \leq v(\mathbf{n})-v\left(\mathbf{n}-\epsilon \mathbf{e}_{t}\right),
\end{aligned}
$$

where $8(i i)^{\prime}$ holds with equality for an active profile $\mathbf{n}$ and $\epsilon$ is an infinitesimal mass. Note that for the left inequality in $8(i i i)^{\prime}$ we assume that $\mathbf{n}+\epsilon \mathbf{e}_{t} \in A$, and, similarly, we assume $\mathbf{n}-\epsilon \mathbf{e}_{t} \in A$ with respect to the inequality on the right. Then, $8(i i i)^{\prime}$ boils down to the neoclassical optimality condition that equates the marginal productivity of factor $t$ to its reward, $\partial v(\mathbf{m}) / \partial m_{t}=x_{t}^{1}$, when evaluated at an active profile $\mathbf{n}$.

When we combine the properties listed in Theorem 2 (ii)-(iii) with the LE payoffs formula (6), we can find explicit expressions for $\mathbf{n}$ and $\mathbf{x}^{1}$ as functions of $v($.$) , bargaining powers \alpha_{1}, \ldots, \alpha_{T}$ and input supplies $N_{1}, \ldots, N_{T}$.

Before we introduce our labor market game, we explain the relationship between our continuous approximation and the discrete game. First, we can approximate the unique LE profile $\mathbf{n}$ in the continuous game with the surplus $v($.$) by a vector \mathbf{n}_{\eta}$ of non-negative numbers with $\eta$ digits after the decimal point. Then, the integer profile $10^{\eta} \mathbf{n}_{\eta}$ in a (large) discrete game with the surplus $v_{\eta}(\mathbf{m})=v\left(10^{-\eta} \mathbf{m}\right)$ will approximately satisfy the LE conditions 2(ii)-(iii). Note that we can interpret $10^{\eta}$ as units (e.g., thousands if $\eta=3$ ) in which player numbers are measured. In the last paragraph of the following example, we show also that the unique active profile $\mathbf{n}$ in the continuous game narrows down the active profile in the discrete game with the same function $v($.$) . The latter profile will be computed by rounding \mathbf{n}$ to an integer vector.

We next turn to the application of our theory to the study of a labor market. In doing so we build on the literature on search-theoretic labor market models. We follow in the footsteps of Delacroix (2003) by modeling the labor market as a matching between heterogeneous agents with transferrable utility. Unlike Delacroix (2003), where a firm is modeled as the matching of two partners, in our framework a firm size in terms of its various factors of production is endogenously determined. In this respect, our work is related to Stole and Zwiebel (1996a) and Stole and Zwiebel (1996b). Our bargaining procedure differs from the one studied by these two authors. In particular, whereas the threat points of the workers in Stole and Zwiebel $(1996 a, b)$ are exogenously given, they are determined endogenously in our model. The implications that we derive with regards to wages and firm size differ therefore, not surprisingly, from those of Stole and Zwiebel (1996a,b). In this respect, our model is similar in spirit to that of Westermark (2003). A crucial difference between this work and that of Westermark (2003), however, is that while we study multilateral bargaining, wages are determined by a sequence of bilateral bargaining events in his model.

Next we present our labor market game. The labor market consists of $T$ types of workers (factors of production), with the mass of workers of type $t=1, \ldots, T$ equal to $N_{t}$, and a unit mass $N_{0}=1$ of equity 
holders (type 0). The equity holders own the Cobb-Douglas production function,

$$
v(\mathbf{z})=c \prod_{t=1}^{T} z_{t}^{\gamma_{t}}, \quad c>0, \gamma_{t}>0, \quad \sum_{t=1}^{T} \gamma_{t}<1
$$

that maps the profile $\mathbf{z}=\left(z_{1}, \ldots, z_{T}\right)$ of worker types into a surplus. Note that this function is strictly concave as the sum of the output elasticities $\gamma_{t}$ is less than one. First we establish that the unique LE $\mathbf{x}^{1}$ is partitioning by showing that there is a unique active profile $\mathbf{n}=\left(n_{1}, \ldots, n_{T}\right)$. For the sake of contradiction, suppose there are two distinct active profiles $\mathbf{n}, \mathbf{m}$. As these profiles are active they satisfy $8(i i)^{\prime}$ with equality,

$$
v(\mathbf{n})=\sum_{t=1}^{T} n_{t} x_{t}^{1}+x_{0}^{1}, \quad v(\mathbf{m})=\sum_{t=1}^{T} m_{t} x_{t}^{1}+x_{0}^{1}
$$

Next consider the profile $(\mathbf{n}+\mathbf{m}) / 2 \in A$ and notice that

$$
v\left(\frac{\mathbf{n}+\mathbf{m}}{2}\right)>\frac{1}{2} v(\mathbf{m})+\frac{1}{2} v(\mathbf{n})=x_{0}^{1}+\sum_{t=1}^{T} \frac{n_{t}+m_{t}}{2} x_{t}^{1} \geq v\left(\frac{\mathbf{n}+\mathbf{m}}{2}\right),
$$

where the first inequality follows from the strict concavity of $v$ and the second from $8(i i)^{\prime}$. This establishes the contradiction.

Given the unique active profile $\mathbf{n}$, the corresponding PE payoffs defined in (6), imply that the relative limit payoffs of different types satisfy,

$$
\frac{x_{t}^{1}}{x_{s}^{1}}=\frac{n_{t} \alpha_{t} N_{s}}{n_{s} \alpha_{s} N_{t}}, \quad s, t=0, \ldots, T .
$$

The latter relationship reveals that the relative scarcity of each type in the market influences the relative wages in the expected way: a higher supply $N_{t}$ of type $t$ depresses their relative individual wages ceteris paribus. On the other hand, the mass $n_{t}$ of workers of type $t$, employed in PE, has a positive impact on their relative wages. This is not surprising, when we observe that $n_{t}$ represents the demand for this type by the equilibrium firm. Therefore, (9) combines in a clear-cut manner the supply and demand forces in the market, adjusted by the type-specific bargaining power.

For the sake of notational simplicity, we assume now equal bargaining powers for each group in the PE profile,

$$
n_{s} \alpha_{s}=n_{t} \alpha_{t}, \quad \text { for all } t, s=0, \ldots, T .
$$

First, we compute the unique PE payoffs for all worker types, given the profile $\mathbf{n}$. By $8(\text { iii })^{\prime}$,

$$
x_{t}^{1}=\frac{\partial v(\mathbf{n})}{\partial n_{t}}=\frac{\gamma_{t}}{n_{t}} v(\mathbf{n}) \text { for all } t=1, \ldots, T,
$$

which entails that the residual payoff to the equity holders can be calculated as,

$$
\begin{gathered}
v(\mathbf{n})=x_{0}^{1}+\sum_{t=1}^{T} n_{t} x_{t}^{1}=x_{0}^{1}+\sum_{t=1}^{T} \gamma_{t} v(\mathbf{n}) \Rightarrow x_{0}^{1}=\gamma_{0} v(\mathbf{n}), \\
\text { where } \gamma_{0}=1-\sum_{t=1}^{T} \gamma_{t} .
\end{gathered}
$$

Using (11), the limit payoffs (6), and the equal bargaining power assumption (10), we can derive the quantity of factor inputs and factor payoffs in an active coalition,

$$
n_{t}=\frac{N_{t} \gamma_{t}}{\gamma_{0}}, \quad x_{t}^{1}=\frac{c}{N_{t}} \prod_{s=0}^{T}\left(N_{s} \gamma_{s}\right)^{\gamma_{s}}, \quad \text { for } t=1, \ldots, T
$$


In this PE, an increase in the productivity of type $s$ leads to higher employment levels and higher payoffs for all types of workers,

$$
\frac{\partial n_{t}}{\partial \gamma_{s}}>0, \quad \frac{\partial x_{t}^{1}}{\partial \gamma_{s}}>0, \quad \frac{\partial x_{0}^{1}}{\partial \gamma_{s}}>0, \quad \text { for all } t, s=1, \ldots, T .
$$

On the other hand, increasing the supply of type $s$ decreases the payoff of this type, while it increases the demand for it and the payoffs to all other players,

$$
\frac{\partial x_{s}^{1}}{\partial N_{s}}<0, \quad \frac{\partial n_{s}}{\partial N_{s}}>0, \quad \frac{\partial x_{t}^{1}}{\partial N_{s}}>0, \quad \frac{\partial x_{0}^{1}}{\partial N_{s}}>0, \quad t, s=1, \ldots, T, t \neq s .
$$

Notice that in the PE discussed above not all coalitions which are matched reach an agreement. Thus, some workers choose to remain unemployed in anticipation of higher wages. This insight complies with the stylized facts found in the search-theoretic literature, cf. Yashiv (2007). We derive these results, however, in an environment where market frictions, as captured by the matching probabilities, do not matter for the equilibrium behavior. Moreover, positive equilibrium level of unemployment co-exists with the neoclassical competitive equilibrium rule that rewards all inputs their marginal product.

In order to compare the results in the continuous approximation and in the discrete game, we consider the case $T=1$ (one worker type). Then, (12) simplifies to,

$$
n_{1}=\frac{N_{1} \gamma_{1}}{1-\gamma_{1}}, \quad x_{1}^{1}=\frac{c\left(N_{1} \gamma_{1}\right)^{\gamma_{1}}\left(1-\gamma_{1}\right)^{1-\gamma_{1}}}{N_{1}}, \quad x_{0}^{1}=N_{1} x_{1}^{1} .
$$

The results for the discrete game, on the other hand, obtain as the limit $\delta \rightarrow 1$ solution to the system,

$$
\begin{aligned}
& x_{0}=\delta x_{0}+\sum_{n=1}^{N_{1}} \frac{1}{N_{1}} \max \left(\frac{n^{\gamma_{1}}-\delta x_{0}-n \delta x_{1}}{2}, 0\right), \\
& x_{1}=\delta x_{1}+\sum_{n=1}^{N_{1}} \frac{1}{N_{1}} \frac{\left(\begin{array}{c}
N_{1}-1 \\
n-1
\end{array}\right)}{\left(\begin{array}{c}
N_{1} \\
n
\end{array}\right)} \max \left(\frac{n^{\gamma_{1}}-\delta x_{0}-n \delta x_{1}}{2 n}, 0\right) .
\end{aligned}
$$

The latter system implies that a coalition composed of one equity owner (type 0 ) and $n=1, \ldots, N_{1}$ workers meets with probability $1 / N_{1}$ and all workers have the same probability to be part of this coalition. For example, for $N_{1}=10, \gamma_{1}=0.4$ and $c=1$, we obtain $n_{1}=6.667, x_{1}^{1}=0.128$ and $x_{0}^{1}=1.281$ from (13) and $x_{1}=0.128, x_{0}=1.281$ from (14) when $\delta=0.99999$. The latter results imply that $n^{\gamma_{1}}-\delta x_{0}^{1}-n \delta x_{1}^{1}$ is non-negative for $n=7$ only. Hence, only coalitions with 7 workers will agree in the discrete game.

\section{Appendix}

For the sake of clarity we recall the shorthand notation $x(S)=\sum_{i \in S} x_{i}$ that will be used in all the analysis that follows.

Lemma 1 If $\delta_{k}=\delta$ for all $i \in \mathcal{N}$, then there is a threshold $\underline{\delta}<1$ such that for all $\delta \in(\underline{\delta}, 1)$ the set of coalitions $\{S \subseteq \mathcal{N}: v(S)>$ $\left.\delta x^{\delta}(S)\right\}$ is the same and the $\mathbf{x}^{\delta}$ converges to a finite limit $\mathbf{x}^{1}$ as $\delta \rightarrow 1$.

Proof 1 In Theorem 1, we show that there is a unique solution $\mathbf{x}^{\delta}$ to (1) for any $\delta_{1}=\ldots=\delta_{N}=\delta \in(0,1)$. Let $\theta(S)$ be the agreement probability for the coalition $S$ in the $L E \mathbf{x}^{\delta}$, i.e., $\theta(S)=1$ if $v(S) \geq \delta x^{\delta}(S)$ and $\theta(S)=0$ if $v(S)<\delta x^{\delta}(S)$. We replace the max operators in (1) by the probabilities $\boldsymbol{\theta}=\{\theta(S)\}_{S \subset \mathcal{N}}$. This converts (1) into a linear system with the unique solution $\mathbf{x}^{\delta}$. This solution is given by the Cramer's rule and equals the ratio of two determinants that are polynomials in $\delta$ of degree at most $N$,

$$
x_{i}^{\delta}=P_{i}(\boldsymbol{\theta}, \delta) / Q(\boldsymbol{\theta}, \delta) .
$$


Note that $Q_{i}(\boldsymbol{\theta}, \delta) \neq 0$ for all $\delta \in(0,1)$ as the system (1) is nonsingular. By the same argument as in the proof of Proposition 1 in Manea (2011), it follows then that,

(F1) The set $\bar{\Delta}$ of $\delta$ for which there is a coalition $S \subseteq \mathcal{N}$ such that $\delta x^{\delta}(S)=v(S)$ contains a finite number of elements.

We define $\underline{\delta}=\max \bar{\Delta}$. Then, the function $v(S)-\bar{\delta} x^{\delta}(S)$ is continuous in $\delta \in(\underline{\delta}, 1)$ as it is a rational function (ratio of two polynomials in (15)) and it has no roots outside $\bar{\Delta}$ by $(F 1)$. By Theorem 1 , for any $\delta^{0}>\underline{\delta}$ there is a unique solution $\mathrm{x}^{\delta^{0}}$ to (1), which implies the agreement probabilities $\boldsymbol{\theta}^{0}=\left\{\theta^{0}(S)\right\}$. By the same argument as in the proof of Proposition 2 in Manea (2011) the following claims follow.

$(F 2)$ For all $\delta>\underline{\delta}, v(S)>\delta x^{\delta}(S)$ if $\theta^{0}(S)=1$ and $v(S)<\delta x^{\delta}(S)$ if $\theta^{0}(S)=0$. The vector $\boldsymbol{\theta}^{0}$ defines the limit cooperation structure.

(F3) The vector of payoffs $\mathbf{x}^{\delta}$ converges to a finite limit $\mathbf{x}^{1}$ as $\delta \rightarrow 1$.

Lemma 2 In the $P E \mathbf{x}^{1}$,

$$
\forall S, S^{\prime} \subseteq \mathcal{N}: \boldsymbol{T}(S) \neq \boldsymbol{T}\left(S^{\prime}\right), S \cap S^{\prime} \neq \varnothing, \quad x^{1}(S)=v(S) \Rightarrow x^{1}\left(S^{\prime}\right)>v\left(S^{\prime}\right) .
$$

Proof 2 If $x^{1}(S)=v(S)$ and $x^{1}\left(S^{\prime}\right) \leq v\left(S^{\prime}\right)$ then, by the definition (5), $\boldsymbol{T}(S)=\boldsymbol{T}\left(S^{\prime}\right)$, which contradicts the statement $\boldsymbol{T}(S) \neq \boldsymbol{T}\left(S^{\prime}\right)$.

Lemma 3 If the $S E \mathbf{x}^{\delta}$ (where $\boldsymbol{\delta}=(\delta, \ldots, \delta)$ ) implies that all players of types $t$ and $s$ cooperate only in coalitions of type $\mathbf{n}=$ $\left(n_{1}, \ldots, n_{T}\right)$, then,

$$
n_{s} \alpha_{s} x^{\delta}\left(\mathcal{N}_{t}\right)=n_{t} \alpha_{t} x^{\delta}\left(\mathcal{N}_{s}\right)
$$

Proof 3 By summing up (1) over all t-type players for some $t \in\{1, \ldots, T\}$, one obtains the total payoff to players of this type,

$$
\begin{aligned}
x^{\delta}\left(\mathcal{N}_{t}\right) & =\delta x^{\delta}\left(\mathcal{N}_{t}\right)+n_{t} \alpha_{t} \sum_{S: \boldsymbol{T}(S)=\mathbf{n}} \pi_{S} \frac{v(S)-\delta x(S)}{\alpha(S)} \\
& =\frac{n_{t} \alpha_{t}}{1-\delta} \sum_{S: \boldsymbol{T}(S)=\mathbf{n}} \pi_{S} \frac{v(S)-\delta x(S)}{\alpha(S)}=: \frac{n_{t} \alpha_{t}}{1-\delta} \Delta^{\delta}(\mathbf{n}) .
\end{aligned}
$$

By the same argument, the total payoff to the players of type $s \in\{1, \ldots, T\}$ is $x^{\delta}\left(\mathcal{N}_{s}\right)=n_{s} \alpha_{s} \Delta^{\delta}(\mathbf{n}) /(1-\delta)$ and the claim follows.

\section{Proof 4 Theorem 2:}

(i) First, we show that $v(S) \leq x^{1}(S)$ for each coalition $S \subseteq \mathcal{N}$ in the $L E \mathbf{x}^{1}$. If there existed a coalition $S$ such that $v(S)>$ $x^{1}(S)$, then $\pi_{S}>0$ due to our assumption in Section 4 and, by re-arranging (1) for a player $i \in S$ and taking the limit, we would obtain a contradiction,

$$
\underbrace{\lim _{\delta \rightarrow 1}(1-\delta) x_{i}^{\delta}}_{=0}=\underbrace{\lim _{\delta \rightarrow 1} \sum_{S \in \mathcal{S}_{i}} \pi_{S} \frac{\alpha_{i}}{\alpha(S)} \max \left\{v(S)-\delta x^{\delta}(S), 0\right\}}_{>0},
$$

where $\delta=\delta_{1}=\ldots=\delta_{N}$. Furthermore, if $S$ is active in the $L E \mathbf{x}^{1}, v(S)>\delta x^{\delta}(S)$ for all $\delta<1$ sufficiently close to one (Lemma 1) and, hence, (16) implies $v(S)=x^{1}(S)$ for each active coalition $S$ in the $L E \mathbf{x}^{\mathbf{1}}$.

Now, in order to prove LE payoff equality for players of the same type, we assume, for the sake of contradiction, that $x_{i}^{1}>x_{j}^{1}$ for two distinct players of the same type, $\boldsymbol{T}(\{i\})=\boldsymbol{T}(\{j\})$, with $i \in S$ and $j \notin S$ for a coalition $S$ that is active in the LE $\mathbf{x}^{\mathbf{1}}$. If $S^{\prime}:=\{j\} \cup S \backslash\{i\}$, then $\boldsymbol{T}(S)=\boldsymbol{T}\left(S^{\prime}\right)$ and,

$$
x^{1}(S)=v(S)=v\left(S^{\prime}\right)>x^{1}\left(S^{\prime}\right)=x^{1}(S)-x_{i}^{1}+x_{j}^{1},
$$

which contradicts that $v\left(S^{\prime}\right) \leq x^{1}\left(S^{\prime}\right)$ for any coalition $S^{\prime} \subseteq \mathcal{N}$ in the $L E \mathbf{x}^{\mathbf{1}}$. Hence, we conclude that $x_{i}^{1}=x_{j}^{1}$ if $\boldsymbol{T}(\{i\})=$ $\boldsymbol{T}(\{j\})$.

(ii) We showed that $\mathbf{x}^{1}(S) \geq v(S)$ (with equality of active coalitions) and that all players of the same type receive equal limit payoffs in (i). From these results, it follows directly that $\sum_{t} n_{t} x_{t}^{1}=v(\mathbf{n})$ for each active coalition of type $\mathbf{n}=\left(n_{1}, \ldots, n_{T}\right)$. 
(iii) Consider a player of type $t$ who cooperates in the $L E \mathbf{x}^{1}$ in a coalition $S$ with $\boldsymbol{T}(S)=\mathbf{n}$. Then, by (i), we can write

$$
x^{1}(S)=v(S)=v(\mathbf{n})=\sum_{t=1}^{T} n_{t} x_{t}^{1}, \quad x^{1}\left(S^{\prime}\right) \geq v\left(S^{\prime}\right), \quad \forall S^{\prime} \subseteq \mathcal{N} .
$$

The latter inequality must hold, in particular, for

$$
S^{\prime}=S^{-}: \boldsymbol{T}\left(S^{-}\right)=\mathbf{n}-\mathbf{e}_{t} \quad \& \quad S^{\prime}=S^{+}: \boldsymbol{T}\left(S^{+}\right)=\mathbf{n}+\mathbf{e}_{t} .
$$

whenever $v\left(\mathbf{n}-\mathbf{e}_{t}\right)$ and $v\left(\mathbf{n}+\mathbf{e}_{t}\right)$ are well-defined values. Then,

$$
\begin{aligned}
& x^{1}\left(S^{-}\right)=\sum_{s \neq t} n_{s} x_{s}+\left(n_{t}-1\right) x_{t}=v(\mathbf{n})-x_{t} \geq v\left(\mathbf{n}-\mathbf{e}_{t}\right)=v\left(S^{-}\right), \\
& x^{1}\left(S^{+}\right)=\sum_{s \neq t} n_{s} x_{s}+\left(n_{t}+1\right) x_{t}=v(\mathbf{n})+x_{t} \geq v\left(\mathbf{n}+\mathbf{e}_{t}\right)=v\left(S^{+}\right),
\end{aligned}
$$

which yields the claim.

Proof 5 Theorem 3:

(i) To show that in the PE, all players of the same type cooperate in MCs of homogeneous types, we will proceed by establishing a contradiction. Consider the $P E \mathbf{x}^{1}$ and assume, for the sake of contradiction, that in $\mathbf{x}^{1}$ players $i$ and $j$ of type $t$ cooperate in coalitions $S$ and $S^{\prime}$, respectively, with $\boldsymbol{T}(S)=\left(n_{1}, \ldots, n_{T}\right) \neq\left(n_{1}^{\prime}, \ldots, n_{T}^{\prime}\right)=\boldsymbol{T}\left(S^{\prime}\right)$. Let the coalition $S^{\prime \prime}$ be the same as $S^{\prime}$ except for player $i$ who replaces player $j$, i.e. $S^{\prime \prime}=S^{\prime} \cup\{i\} \backslash\{j\}$. Hence, $\boldsymbol{T}(S) \neq \boldsymbol{T}\left(S^{\prime}\right)=\boldsymbol{T}\left(S^{\prime \prime}\right)$ and $\{i\} \in S \cap S^{\prime \prime}$. As $S$ and $S^{\prime}$ are active, Theorem 2, items (i) and (ii), and the fact that $\boldsymbol{T}\left(S^{\prime}\right)=\boldsymbol{T}\left(S^{\prime \prime}\right)$ imply,

$$
v(S)=x^{1}(S), \quad x^{1}\left(S^{\prime}\right)=v\left(S^{\prime}\right)=v\left(S^{\prime \prime}\right)=x^{1}\left(S^{\prime \prime}\right) .
$$

However, this contradicts, by Lemma 2, the partitioning property of $\mathbf{x}^{\mathbf{1}}$ as $S \cap S^{\prime \prime} \neq \varnothing$ and $\boldsymbol{T}(S) \neq \boldsymbol{T}\left(S^{\prime \prime}\right)$.

(ii) Let $\delta=\delta_{1}=\ldots=\delta_{N}$ and consider the PE $\mathbf{x}^{\mathbf{1}}$. We have established that each player of type $t$ cooperates in MCs of homogeneous types. By Lemma 3, the total payoff for all players of type t and all players of type s, that cooperate in MCs of the same type as a type $t$-player, i.e., $\mathbf{n}=\left(n_{1}, \ldots, n_{T}\right)$, satisfy

$$
n_{s} \alpha_{s} x^{\delta}\left(\mathcal{N}_{t}\right)=n_{t} \alpha_{t} x^{\delta}\left(\mathcal{N}_{s}\right) .
$$

This equality must hold also for the PE $\mathbf{x}^{1}$. Thus using Theorem 2(i), we can re-write the last equality as

$$
n_{s} \alpha_{s} N_{t} x_{t}^{1}=n_{t} \alpha_{t} N_{s} x_{s}^{1} .
$$

In particular, $x_{t}^{1}=0$ implies $x_{s}^{1}=0$ for any two distinct types $s$ and that cooperate in a coalition $S: \boldsymbol{T}(S)=\mathbf{n}$. This is only possible in PE if $v(S)=v(\mathbf{n})=0$. Suppose $x_{t}^{1}>0$ for some type $t$. Then, by Theorem 2 items (i) and (ii), it follows that

$$
v(S)=v(\mathbf{n})=x^{1}(S)=\sum_{s=1}^{T} n_{s} x_{s}^{1} .
$$

Using (17) to substitute for $x_{s}^{1}$,

$$
v(\mathbf{n})=\sum_{s=1}^{T} \frac{n_{s}^{2} \alpha_{s} N_{t} x_{t}^{1}}{n_{t} \alpha_{t} N_{s}}=x_{t}^{1} \frac{N_{t}}{n_{t} \alpha_{t}} \sum_{s=1}^{T} \frac{n_{s}^{2} \alpha_{s}}{N_{s}} .
$$

By re-arranging the above expression, we obtain the payoff of each type-t player in the $P E \mathbf{x}^{\mathbf{1}}$,

$$
x_{t}^{1}=v(\mathbf{n}) \frac{n_{t} \alpha_{t} / N_{t}}{\sum_{s=1}^{T}\left(n_{s}^{2} \alpha_{s} / N_{s}\right)} .
$$


Proof 6 Theorem 4

$\mathbf{P E} \Rightarrow(7):$ In a PE, there is a partition $\mathcal{P}$ of player types and all types in the partition element $P \in \mathcal{P}$ cooperate in coalitions of a unique type $\mathbf{n}^{P}$ obtaining payoffs $\left\{x_{t}^{1}\right\}_{t \in P}$ computed by the formula (6). Then, by the LE condition 2(ii),

$$
\begin{gathered}
\sum_{P \in \mathcal{P}} \sum_{t \in P} m_{t} x_{t}^{1}>v(\mathbf{m}), \quad \forall \mathbf{m} \notin\left\{\mathbf{n}^{P}\right\}_{P \in \mathcal{P}}, \mathbf{m} \neq \mathbf{0} . \\
\text { where, } \quad x_{t}^{1}=\frac{v\left(\mathbf{n}^{P}\right) n_{t}^{1} \alpha_{t} / N_{t}}{\sum_{s \in P}\left(\alpha_{s} n_{s}^{2} / N_{s}\right)}, \quad t \in P .
\end{gathered}
$$

We observe that the condition (18) is equivalent to (7).

$(7) \Rightarrow$ PE: The condition (7) can be written as,

$$
\begin{gathered}
\sum_{P \in \mathcal{P}} \sum_{t \in P} m_{t} x_{t}^{1}>v(\mathbf{m}), \quad \forall \mathbf{m} \notin\left\{\mathbf{n}^{P}\right\}_{P \in \mathcal{P}}, \mathbf{m} \neq \mathbf{0} . \\
\text { where, } \quad x_{t}^{1}=\frac{v\left(\mathbf{n}^{P}\right) n_{t}^{1} \alpha_{t} / N_{t}}{\sum_{s \in P}\left(\alpha_{s} n_{s}^{2} / N_{s}\right)}, \quad t \in P .
\end{gathered}
$$

On the other hand,

$$
\sum_{t=1}^{T} \mathbf{n}_{t}^{P} x_{t}^{1}=\sum_{t \in P} \frac{v\left(\mathbf{n}^{P}\right) n_{t}^{2} \alpha_{t} / N_{t}}{\sum_{s \in P}\left(\alpha_{s} n_{s}^{2} / N_{s}\right)}=v\left(\mathbf{n}^{P}\right), \quad P \in \mathcal{P} .
$$

Hence, $\left\{\mathbf{n}^{P}\right\}_{P \in \mathcal{P}}$ are the only productive profiles that satisfy the LE condition $2(i i)$ with equality when the LE payoffs are computed by the formula (6). Therefore, $\left\{\mathbf{n}^{P}\right\}_{P \in \mathcal{P}}$ are the only active profiles. We note further that any coalition of type $\mathbf{n}^{P}$ has no intersection with a coalition of type $\mathbf{n}^{P \prime}$ if $P, P^{\prime} \in \mathcal{P}$ and $P \neq P^{\prime}$. Hence, any two active coalitions intersect only if they are of the same type. We conclude then that the LE is partitioning and the Theorem 3 confirms that the LE payoffs are given by (6).

\section{References}

Binmore, K. and Herrero, M.: 1988, Matching and bargaining in dynamic markets, Review of Economic Studies 55, 17-31.

Chatterjee, K., Dutta, B., Ray, D. and Sengupta, K.: 1993, A noncooperative theory of coalitional bargaining, Review of Economic Studies 60, 463-477.

Chatterjee, K. and Sabourian, H.: 2000, Multiperson bargaining and strategic complexity, Econometrica 68, 1491-1509.

Delacroix, A.: 2003, Heterogeneous matching with transferable utility: Two labor market applications, International Economic Review 44, 313-330.

Duffie, D., Gâarleanu, N. and Pedersen, L.: 2005, Over the counter markets, Econometrica 73, 1815-1847.

Gul, F.: 1989, Bargaining foundations of shapley value, Econometrica 57, 81-95.

Hart, S. and Mas-Collel, A.: 1996a, Bargaining and value, Econometrica 64, 357-380.

Hart, S. and Mas-Collel, A.: 1996b, Harsanyi values of large economies: Nonequivalence to competitive equilibria, Games and Economic Behavior 13, 74-79.

Huang, C.-Y.: 2002, Multilateral bargaining: conditional and unconditional offers, Economic Theory 20, $401-412$.

Krishna, V. and Serrano, R.: 1996, Multilateral bargaining, Review of Economic Studies 63, 61-80.

Manea, M.: 2011, Bargaining in stationary networks, Americal Economic Review 101, 2042-80.

Nguyen, T.: 2011, Coalitional bargaining in networks. Available online http://users.eecs.northwestern.edu/ thanh/paper/bargaining.pdf. Okada, A.: 1996, A noncooperative coalitional bargaining game with random proposers, Games and Economic Behavior 16, $97-108$.

Okada, A.: 2011, Coalitional bargaining games with random proposers: Theory and application, Games and Economic Behavior 73, 227-235

Polanski, A. and Vega-Redondo, F.: 2013, Markets, bargaining, and networks with heterogeneous agents. Working Paper.

Rubinstein, A.: 1982, Perfect equilibrium in a bargaining model, Econometrica 50, 97-109.

Rubinstein, A. and Wolinsky, A.: 1985, Equilibrium in a market with sequential bargaining, Econometrica 53, 1133-1150.

Rubinstein, A. and Wolinsky, A.: 1990, Decentralized trading, strategic behaviour and the walrasian outcome, Review of Economic Studies 57, 63-78.

Siedlarek, J.-P.: 2012, Intermediation in networks, FEEM Working Paper No. 42.2012 .

Stole, L. and Zwiebel, J.: 1996a, Organizational design and technology choice under intrafirm bargaining, American Economic Review 80, 195-222.

Stole, L. and Zwiebel, J.: 1996b, Intra-firm bargaining under non-binding contracts, Review of Economic Studies 63, 375-410. 
Sutton, J.: 1986, Non-cooperative bargaining theory: An introduction, Review of Economic Studies 53, 709-724.

Westermark, A.: 2003, Bargaining, binding contracts and competitive wages, Games and Economic Behavior 43, 296-311.

Yan, H.: 2003, Noncooperative selection of the core, International Journal of Game Theory 31, 527-540.

Yan, H.: 2009, Uniqueness in random-proposer multilateral bargaining, International Game Theory Review 11, 407-417.

Yashiv, E.: 2007, Labor search and matching in macroeconomics, European Economic Review 51, 1859-1895. 